\title{
Factors associated with dietary glycemic index and glycemic load in pregnant women and risk for gestational diabetes mellitus
}

\begin{abstract}
The risk of gestational diabetes mellitus (GDM) increases during the second trimester of pregnancy. However, the role of dietary glycemic index (GI) and glycemic load (GL) on GDM risk is controversial. We aimed to determine the association of established risk factors of GDM with GI and GL among healthy pregnant women, and whether GI and GL were subsequently related to GDM risk. Dietary GI and GL were assessed in healthy pregnant women from the Seremban Cohort Study using a food frequency questionnaire. After adjusting for energy intake, high GI was significantly associated with lower household income, shorter stature, higher proportion of carbohydrate intake, lower sugar proportion and lower fibre intake. High GL was significantly associated with younger maternal age, higher carbohydrate proportion and lower fibre intake. GI and GL intakes were not significantly associated with GDM risk. However, they were associated with a few established risk factors of GDM.
\end{abstract}

Keyword: Gestational diabetes mellitus; Glycemic index; Glycemic load; Diet; Pregnancy 